\title{
Automation of Routine Work: A Case Study of Employees' Experiences of Work Meaningfulness
}

\author{
Anne Staaby \\ Kristiania University College \\ Dept. Technology, Oslo, Norway \\ annestaaby@gmail.com
}

\author{
Kjeld Hansen \\ Kristiania University College \\ Dept. Technology, Oslo, Norway \\ kjeld.hansen@kristiania.no
}

\author{
Tor-Morten Grønli \\ Kristiania University College \\ Dept. Technology, Oslo, Norway \\ tor-morten.gronli@kristiania.no
}

\begin{abstract}
The idea of automation replacing humans in the workplace has been given considerable attention, while less attention has been afforded to how people develop a meaningful work life with automation of routine work. In this study we investigate how employees, who have had their routine work automated with RPA, have experienced its influence on their work and its meaningfulness. Concretely, we conduct a case study of how employees experience this process in three different case organizations in Oslo, Norway. We make theoretical contributions by combining automation of work, RPA and work meaningfulness literature to understand what opportunities and pitfalls the organizations experience, when they seek to harness value from their human resources in the process. We also contribute with implications for practice, by suggesting organizations to focus on creating autonomy and job crafting opportunities for employees, when they automate routine work with RPA.
\end{abstract}

\section{Introduction}

Over the past few years, the use of robotic process automation (RPA) for automation of routine work has grown exponentially in several domains, such as the finance, insurance, logistics, and IT; much due to its promises of increasing efficiency and gaining significant returns on investment [1]. The idea of automation replacing humans in the workplace has been given considerable attention, while less attention has been afforded to how people develop a meaningful work life with automation of routine work [2] [3]. In this study we investigate how employees, who have had their routine work automated with RPA, have experienced its influence on their work and its meaningfulness.

Overall, RPA can be considered a low-level form of intelligent automation, which is suitable for automation of routine work. The field of intelligent automation in information systems (IS) has primarily been driven by computer science and focused on technical capabilities [4] [5], strategic and operational organizational benefits [6], future implications, and normative argumentation, such as intelligent technologies for "good" or for "bad" [7]. There are studies that report on individual level benefits of intelligent automation, but it appear to be an insufficiently researched area [5]. In one study, individual level benefits were not anticipated by neither the case organization nor the investigating researchers, but nevertheless uncovered through their research. Such unexpected benefits include: Improvement in staff knowledge and skills; increase in job satisfaction; and improvement in employee participation in value adding activities [6]. In this study we introduce a sociotechnical perspective as it can broaden the views on intelligent automation in IS through its incorporation of the individual aspect with the technical, social and managerial. The sociotechnical perspective incorporates technical artifacts as well as the individuals using and developing these artifacts in a social context. Ignoring the individual level of intelligent automation could be considered a missed opportunity for reflecting on its consequences and for critiquing a development in practice and research, where there so far has been payed insufficient attention to the human aspect [8]. We intend to focus on the individual level particularly as it can help us to explore the human aspect of intelligent automation [9] [10].

The unexpected benefits reported by Vitheranage et al. [6] arguably have characteristics, which appear to be connected to experiences of meaningful work and the construction of meaningfulness. Smids et al. [2], who have studied robots in the workplace, argue that there are several reasons why it is important to understand how robotization may impact employees' experience of meaningful work. Firstly, employees spend a significant amount of time at work, so if robotization leads to a decrease in experienced meaningful work it will be disruptive to their quality of life. Secondly, meaningful work has a significant impact on job satisfaction and general well-being. Thirdly, there is arguably a societal 
obligation to ensure that we can all live as full and meaningful lives as possible.

In light of the exponential adoption of RPA for automation of routine work in several domains, we find that there is an urgent need to explore how it influences employees' experience of work meaningfulness through the research question:

How does automation of routine work influence employees' experiences of work meaningfulness?

The article is structured as follows: (2) In the second section we clarify key concepts and summarize the literature on automation of routine work with RPA and the construction of work meaningfulness. (3) In the third section we outline our inductive research design and methodological approach. (4) In the fourth section we present our findings and analytical dimensions. (5) In the fifth section we discuss our key findings and their practical implications. (6) In the sixth section we outline our contributions for theory and practice as well as future perspectives.

\section{Background}

Overall, RPA is considered a process-aware information system and a neighbouring field to business process automation (BPA). Where BPA is often seen as a holistic software platform used for end-to-end processes, and to manage humans, robots and system integration, RPA is used for repetitive sequences of tasks, previously carried out by a human, but now fully delegated to software robots [5]. Because RPA processes are more closely linked with individual employees' work processes than BPA, it is more relevant to discuss this form of automation in relation to work meaningfulness.

\subsection{Automation of work with RPA}

In the emerging literature body, there has been suggested several definitions of RPA [4] [5] [6]. We apply a definition used by Syed et al, which states that RPA is: "[...] a relatively new technology comprising software agents [...] that mimic the manual path taken by a human through a range of computer applications when performing certain tasks in a business process" [5, p. 1]. In general, there are two types of software robots; the rule-based and the intelligent. Rule-based software robots perform structured, repetitive, high volume, high frequency manual tasks, while intelligent software robots can be trained with data and adapt to situations [11] [6]. In this study, we look at low-level intelligent software robots.

To this date, literature on software robots and RPA is largely focused on implementation, organization and use. Because software robots and RPA is a fairly new research stream within IS research, previous studies have mainly been concerned with exploring RPA at an organizational level: demonstrating use cases and effects of RPA [12]; identifying "best practice" and providing guidelines for implementing RPA in organizations $[1,13,12]$; and investigating how RPA should be organized and governed [5, 4]. It has been demonstrated that automation of routine work with RPA can drastically improve the performance of organizations, by increasing compliance, improving service and quality, lowering costs and reducing delivery time [1]. This may explain why many organizations are fascinated by RPA. Furthermore, it can also allow employees to take on tasks that are more fulfilling, sophisticated, and better suited to human strengths [13], and thus more satisfactory for employees. We have identified only a few studies of automation of routine work with RPA, where employees' work has gained new features and characteristics that are consistent with experiences of meaningful work $[6,1]$. In general, the literature identifies two different levels of capabilities associated with RPA; the capabilities that focus on the individual employee and those that focus on the general organization and its processes [13] [5]. Additionally, Syed et al note two types of employee level capabilities associated with RPA: The capability of RPA to change the nature of work and the creation of new roles for employees [5]. In automation of work literature, there are some signs of interest in this topic, such as Sampath and Khargonekar, who encourage organizations to develop a socially responsible automation approach [14]. Such observations further underline the possibility for developing perspectives of work meaningfulness in connection with automation of routine work with RPA. Automation of routine work with RPA can cause changes to work and workplaces, and lead to changes in organizational structures and redesigned roles $[1,5]$. Depending on the work context and procedures, these changes can be experienced as making work more or less meaningful for employees. Generally speaking, people will find their work more meaningful to the extent that RPA complement or elevate their skills, and less so when it substitutes or debilitate skills [15] [2].

\subsection{Work Meaningfulness}

Work meaningfulness can be considered an inter-disciplinary research topic within psychology, sociology, economy and organizational research to name a few [16]. Generally speaking, meaningful work is largely conceptualized as a positively valenced construct [15] and can be defined as "work that is 
experienced as particularly significant and holding more positive meaning for individuals" [16, p. 95]. For employees, experiences of work meaningfulness can be derived from both conducting work-related activities that are found enjoyable and personally enriching, where one is able to express one's full potential (i.e. self-realization), as well as pursuing a purpose and contributing to something beyond pure self-interest [16]. Meaningful work, though it has been categorized in multiple, overlapping and closely connected ways across literature, can be categorized in terms of work dimensions and sources of meaningful work [10]. As an example, Bailey et al. categorize dimensions of meaningful work into four domains; work tasks, roles, the organization, and interaction, while Smids et al. classify sources of meaningful work as pursuing a purpose, social relationships, exercising skills and development, self-esteem and recognition, and autonomy. [17] [2]

Several models suggest that experiences of work meaningfulness is connected with the characteristics of work. According to Hackman and Oldham's well-known "Job Characteristic Theory" experienced meaningfulness of work can arise from the level of autonomy, skill variety, task identity and task significance in one's job [18]. The presence of these characteristics is found to be associated with higher degrees of motivation, satisfaction and performance, which is why recommendations encourage organizations to design jobs accordingly, and facilitate meaningfulness by focusing on enriching the work of employees $[18,19]$.

Some organizational psychologists emphasize the importance of autonomy in experienced work meaningfulness (i.e. [10]). Having freedom in the workplace, being able to participate in work-related decisions, and having opportunities to shape one's work, all represent important aspects of autonomy and personal agency in work [16]. The opportunity to shape one's work is conceptualized in organizational psychology as "job crafting"; Employees do not merely impute meaning from given work characteristics related to job design (provided by management), but also actively shape the meaning of their work by crafting or redesigning work tasks and/or relational boundaries to better align with their preferences, skills, values and personal goals $[20,21]$. It is found that by proactively crafting the job resources and demands, workers experience a higher degree of work meaningfulness, not only directly, but also indirectly through the optimization of person-job fit. When provided with sufficient autonomy to meet their psychological and basic needs, employees have the opportunity to create their own meaningfulness without having the need for organizations to create it for them [10].

As definitions of meaningful work implies, the experienced significance and purpose of one's work plays an important role in the construction of work meaningfulness. Through a sense of purpose, individuals have the ability to link present actions to future desired anticipated states and events [16]. Correspondingly, in the context of work, making a contribution while pursuing a purpose is regarded as making work more meaningful [2]. Work is a context where many individuals have opportunities for excelling and developing new skills. When employees see themselves learning, growing and responding to challenges, they are likely to feel more efficacious and competent (i.e. being able to act and realize certain things) in their work [16]. This, in turn, affords employees with a sense of meaningfulness. Lysova et al. suggest that organizations should enable development opportunities to further provide employees with opportunities to experience work meaningfulness [10]. Work meaningfulness can also arise from self-esteem and social recognition. When workers develop skills, achieve accomplishments or make significant contributions to the organization, their self-esteem increases as they assess themselves more positively and build self-worth [16, 2]. Such experiences can be enhanced through recognition from other individuals or groups, such as managers or colleagues. Such affirmations of success and value strengthen employees' perceptions of the value and meaningfulness of their work, as they enable individuals to strengthen and justify positive views of the self, which is a strong motivational factor for humans [16].

Meaningful work experiences are not constructed in a vacuum [16]. Individuals are constrained by the social context, which consist of expectations that define appropriate behavior, and thus form how they interpret and explain event and actions [19,3]. For employees, organizations are considered very strong social contexts with unique systems of meaning, shaped by the organizational culture, policies and practices, and the organizational leadership [10]. Consequently, the accounts (in this context, the "raw materials" employees use to justify the worth of their work and its meaningfulness) employees create about their job, are heavily influenced by the organizational context, which frames what is valued in the organization $[19,16]$. Framings are a central mechanism applied to examine cultural processes. It focuses on how information is communicated and constructed to affect the motivations, beliefs and actions of others [19]. In the context of work, framings provides the context for employees 
to understand what is valued in their organization, and thus how they justify the value of their work, and thereby, its meaningfulness [3]. Meaningfulness can also arise from interactions and relationships within organizational context. This occurs through a sense of relatedness and belonging [17, 2], which manifest through social identification and interpersonal connectedness with others at work. Because individuals seek to be a part of desired social groups, membership in workplace groups produce a sense of shared common beliefs, identity and attributes that can foster meaningful experiences as they feel like they are a part of something special. [16].

There also exist several barriers to meaningful work, such as the lack of control over, or inability to justify the value of one's work, describing problems related to the feelings of alienation and anomie. Alienation, also referred to as "workers separation", can be explained as the lack of control over one's work. It has also been used to describe the lack of autonomy and constraints in work, which were some of the motivating factors behind Hackman and Oldham's [18] job characteristics model. Work alienation can emerge through certain work conditions, which can produce a lack of meaningfulness, and thus restrain employees from fulfilling their desires, motivations and needs associated with self-realization. Solutions to this problem is largely focused on enriching the work of employees $[19,18]$. The other barrier to meaningful work, the concept of anomie, refers to experiences of uncertainty related to whether one's work holds value [17]. This problem builds on the perspective that the value in one's work, and thus work meaningfulness, is not singlehandedly founded in the design of jobs or certain work tasks, but is something that need to be interpreted, accounted for, and constructed. Correspondingly, as a solution to this problem, literature suggest focusing on employees' account-making activities to provide insights on building meaningful work [19].

\section{Methodology}

This study intends to uncover new insights about an unexplored phenomenon, and thus follows an exploratory research approach. Because routine task automation and its impact on employees' experiences of work meaningfulness is a complex, unstructured and relatively new phenomenon, this study applies a "bottom-up", inductive research approach, focused on discovery and theory building, rather than testing [22] [23] [8] [3] To generate new information and discover new relationships between automation of routine tasks and experiences of work meaningfulness, the study applies a case study strategy, involving data collection from three different case organizations in the public and private sector with offices in Oslo, Norway. The case organizations were selected in accordance with the corpus construction technique in order to ensure a varied and balanced data collection. The strategy was both applied to identify and select case organizations and to identify relevant interviewees within the case organizations [24].

All case organizations had initiated processes of automation of routine work with RPA in 2016 or 2017 and deployed them in 2017 or 2018, which was central to their selection. As previously stated, we wanted to investigate case units within the case organizations that would demonstrate contextual differences when it comes to organizational attributes, in addition to the content of work, as these attributes are relevant to the exploration of work meaningfulness [17]. Thus, an administrative unit in a housing cooperative, an audit unit in a consultancy organization, and a financial unit in a public sector organization were selected as case units. The units were selected in accordance with the case selection criteria, and in cooperation with the case organizations. In all case units, employees already had some experience regarding the implementation and use of RPA. Therefore, the three case units were good choices for exploring the phenomenon. We chose a semi-structured interview approach to allow for discovery and in-depth investigations of how people within the organizations perceive RPA and how it influences their experience of work meaningfulness. The semi-structured interview was considered a suitable approach as it provides some structure, while allowing for exploration and improvisation necessary for an exploratory study [25]. All of the interviews were conducted face to face in Oslo, Norway, which allowed for a higher level of interpersonal interaction and participant observation, which is expected to generate additional layers of data. We used digital audio recording and took notes to capture the interview data. We conducted a total of 14 interviews with (unit level) managers, implementers, and employees within the three case companies during March, April and October 2019. The interviews had a duration between 35 and 55 minutes with an average length of 45 minutes. All interviews were transcribed and analysed with the qualitative data analytics tool, MAXQDA [26] After studying our data carefully (1) the first and second author performed separate initial open coding of the data in line with Corbin and Strauss, Stein et al., and Courpasson and Montries [22] [23] [3]. This resulted in 67 first order codes. (2) We consolidated our first order codes by integrating the first and second authors 


\begin{tabular}{ccc}
\hline Case org. 1: & Case org. 2: & Case org. 3: \\
Admin. unit in & Audit unit in & Financial unit in \\
housing coop. & consultancy & public sector org. \\
\hline Implementer 1A & Implementer 2C & Implementer 3A \\
Manager 1C & Implementer 2A & Manager 3B \\
Employee 1B & Manager 2E & Manager 3D \\
Employee 1D & Employee 2B & Employee 3C \\
& Employee 2C & Employee 3E \\
\hline
\end{tabular}

Table 1. Overview of interviewees.

first order codes. This left us with 45 codes. (3) We used axial coding to categorise our first order codes into six categories. (4) We integrated our coding categories with theoretical categories and further aggregated the theoretical categories into four theoretical dimensions, which are in line with the dimensions of Lysova et al.'s work on meaningful work in organizations [10]. The dimensions are as follows: organizational level, managerial level, job level and employee level. We will use these analytical dimensions to explore how the organizations' automation of routine work with RPA influence employees' experiences of automation of work.

\section{Results and analysis}

Our results suggest that automation of routine work with RPA has the ability to influence experiences of meaningful work - both in work and at work. We will elaborate on the results and our analysis in relation to the organizational-, managerial- job- and employee-level dimensions.

\subsection{Organizational Level}

Our analysis shows that organizational culture has significant influence on employees' experiences of meaningfulness. Employees in case organization 1 and 3, carry out similar work tasks, but have different experiences of work meaningfulness. In case organization 3 the organizational culture is shaped by a strong focus on human resources, while the organizational culture in case organization 1 is shaped by constrained work boundaries, with limited autonomy and less control over one's work, which are similar characteristics to those used to describe alienation. For example, employee 1D described how these work conditions affected her experience of work:

"...we are given very specific tasks. We register changes in ownership. We have to do this and that, so there is not much room for being creative. It is what it is... So, we have to focus on simplifying the tasks we already have [...] and there is not much room to think of anything new" (Employee, 1D).

In case organization 2, the organizational culture and work tasks in were different from the other organizations. Employees were generally better educated, performed more varied and complex work with a higher degree of autonomy. The work was found to be more project based, which enhanced the autonomy of the employees and afforded them more opportunities to engage in job crafting; changing their work and job to further improve their skill sets and career opportunities.

In general, the organizational culture was found to align with the organizations' approach to automation of work, which differed between the organizations. Their main purposes for automation of work were cost-reduction, increased quality of services, and improved employee well-being. Although these purposes were mentioned by all organizations, and their approach therefore might seem similar, it was evident that there were differences; one organization was focused on cost-reduction, while others took a broader approach. For example, by including considerations of performance and workforce development, enrichment, and well-being [14].

"There are several purposes, but the main purpose is to increase the quality of the organization. As you save time when manual tasks are performed by a robot, rather than an employee, you free up capacity for the employee so they can do more interesting tasks, which generate greater value to the customer and the audit services" (Manager, 2A).

Hence, how and to what degree the automation of work with RPA cause changes in work and influence meaningful work is arguably related to the organizations' approach.

The organizations' approach to automation was not only found to shape how RPA change work, but also how RPA was communicated and framed within the organizations. Framings serve as accounts for understanding which type of work that is considered valuable to the organization [19]. Thus, how organizations frame RPA seem to influence employees' construction of work meaningfulness related to changes in work. For example, in case organization 1, RPA was framed as a tool that would reduce organizational costs and create more interesting work for employees; in case organization 2, RPA was framed as a technology that would take reduce stress and heavy workloads for employees; while in case organization 3 it was framed as a tool that would increase the quality of services by enabling employees to focus on more value adding work.

Furthermore, we found that in cases with apparent misalignment between the organization's approach to automation of routine work and their communication (i.e. organizational framing) of it, employees 
experienced feelings related to job insecurity due to problems of justifying the value of their work. Specifically, in organization 1, top-management communicated to employees that automation of routine work would lead to new and more interesting work tasks, but their approach to automation was perceived by employees to focus on efficiency and cost-reduction.

"[Top] management is very clear, that you shouldn't fear for your job, because you will get more interesting tasks. But it remains to be seen as we are not that far in the process. So, we don't feel that we have different and more interesting work tasks yet. So you become a bit unsure. How will it be, if everything becomes digitized?" (Employee, 1C).

Simultaneously, as employees experienced an increasing workload of similar tasks, rather than new tasks, their concerns regarding job loss became apparent. These employees' experiences may manifest in feelings of anomie, as they struggle to justify the value of their work.

\subsection{Managerial Level}

In general employees felt relatively well informed of and included in their organizations' plans for automation of routine work with RPA. However, as noted in the previous quote, they did not feel equally comfortable about it. In case organization 1, other employees felt that working with RPA and operating the software robot provided their work with extra responsibilities and feelings of importance.

"We are divided into different groups, and we are responsible for "Gizmo", as we call him [the RPA robot]. We log-on and pay attention to what he is doing, and his deviations... that is our responsibility... we are five people" (Employee, 1B).

When selecting processes for automation of routine tasks, all the case organizations applied RPA selection criteria in line with the criteria suggested in literature and industry reports. Across the board, case organizations focused on automating repetitive tasks occurring within a work process with high volume and frequency. As RPA require standardization of tasks and processes before automation, processes were often redesigned prior to the deployment. Case organizations on several occasions used this as an opportunity to include perspectives of individual fulfillment, which are less articulated in RPA literature.

The case companies did for example involve employees in the pre-implementation phase of RPA. The organizations used different models for employee involvement, such as RPA teams within the department (case organization 1), employee-driven RPA initiatives (case organization 2), and cross-departmental project groups (case organization 3). Across the board, managers and employees from the departments contributed to the mapping, standardization, and redesign of processes. The way and degree to which employees were involved was found to be determined by the characteristics of their work and their degree of specialization. Specifically, in case organization 3 , managers and employees from the financial unit became involved as they possessed expert knowledge within the financial domain. The managers ability to involve employees in the pre-implementation phase of RPA can be characterized as a facilitation of work meaningfulness, which their employees often succeeded in realizing through their abilities to assume more responsibility and added feeling of importance.

"Most of us are experienced auditors and have done similar work. We have a lot of experience with the work that is done here, so we know what can be automated and what needs to be automated. Yes, it is easy for us to identify this type of work" (Implementer, 2A).

Even though the main purpose of involving employees were often related to enabling automation of processes, it could also facilitate process-innovation and engagement related to identifying new opportunities for automation. This could further accelerate the development of organization-wide process automation capabilities.

\subsection{Job level}

The introduction of RPA can lead to changes in work, which makes it necessary to redefine jobs including their qualities and responsibilities. Across the board, employees and managers in the organizations, have all experienced changes in work as a result of automation. These changes relate to two levels; changes in work tasks and changes in interpersonal working relationships inside and outside of the organization. For example, several employees have become involved in RPA-related tasks forces, working with employees and managers from other departments and offices than their own. Such changes contribute to meaningful work experiences for employees. For example, through exercising skills and self-development, pursuing a purpose, increased autonomy, self-esteem and recognition, and strengthened social relationships. Several of these experiences were particularly evident in case organization 2, where automation of routine tasks resulted in an accelerated skill development process. This allowed highly educated, but inexperienced, junior accountants to spend more time on relevant, 
challenging tasks, along with learning and practicing audit methodology:

“... when newly hired first-year employees don't have to spend time on boring, repetitive tasks, they have time to engage in tasks that a more senior person usually would have done. [...] And then everything will shift, so that I, on my level, do more tasks that was previously done by the person above me" (Employee, 2B).

Similarly, in case organization 2, experienced accountants found time to focus on more complex and challenging parts of the audit, such as analyzing numbers and relations, and investigating abnormalities discovered during the audit process: “... we can spend time looking only at the final numbers, and spend time on abnormalities; "Here I spot an abnormality, what is the reason for this?" We can go more in depth; communicate with the customer, [...] I think the customer also experience that we get a better focus and ask the right questions because... suddenly there is more time to look into abnormalities" (Employee, 2C).

As such, we found that RPA enables employees to engage in more enriching work tasks, where they gain valuable experiences and make a significant contribution to their organization and its clients, and thus enhance their self-esteem, which is an important source of work meaningfulness. This also resulted in higher degrees of human interactions at work and detail-oriented interactions with the customer.

"I have more time to sit and talk with clients, and that tends to be where you find things out [...] you spend less time staring at the computer and more time communicating with the clients" (Employee, 2C).

This was found to increase the quality of the audit and strengthening the relationship with the customer.

Across the board, we also found interactions to increase inside the organizations, which often led to new ways of collaborating. Specifically, in case organization 3, involvement in the RPA initiative led to more collaboration across units and the formation of specialized teams within the organization.

This year, our digitization group was tasked with the responsibility to follow-up on deviations and log-in the robot. It's a bit more hands-on. We have our own mail for recording deviations [...] so if the robot stop by given criteria, we have to handle it manually. And we will have to sort out what went wrong, report back to the [IT] team and they will have to change the parameters. They have to train the robot (Employee, 1D).

In some cases, automation of routine work was also found to increase the workload for employees. "Not new types, but more work [...] Now we have been asked to do an increased amount of the same type of work as before ... received from regional offices [...] So we have a higher workload, but not so many new tasks..." (Employee 1C).

In all organizations, RPA was found to cause changes both at work and in work, which again influence employees' experiences of work meaningfulness. It became evident that employees were experiencing the automation of work with both positive and negative outcomes.

\subsection{Employee level}

We found that employees' abilities to seize opportunities for work meaningfulness was clearly linked to the level of autonomy and agency in their jobs. Across the board, employees in the companies were offered and saw opportunities for engaging in more interesting work. However, these opportunities were not realized to the equal extent. One important reason for this was the differences in work autonomy and agency experienced by employees. Specifically, in case organization 2, employees seized the opportunities for shaping their work (i.e. job crafting), by developing and integrating IT skills as a core component in their jobs. For example, employee $2 \mathrm{C}$ invested the time gained through automation to develop programming skills, which he applied to further develop digital solutions for accounting practices within the department.

"At first, I was really just working with accounting and auditing... and that was fine. Later, I was given the opportunity to start in a newly established unit and started to learn programming" (Implementer, 2A).

Such job-crafting activities allow employees to shape the content of their work to better align their interests with their career goals. Hence, creating a better person-job fit [10]. We also found examples of experiences which limit the possibilities for crafting meaningful work experiences. Across the board, the processes of standardization were experienced as a double-edged sword; liberating and limiting at the same time. As previously explained, standardization can be experienced as a time liberating measure. However, it is less evident how standardization can also negatively influence the autonomy of work, in the form of work alienation. For example, manager $2 \mathrm{E}$ explains how his organization balance on this swords edge.

"You have a business and a lot of knowledge workers. They want to work autonomously; to be, to think for themselves, and this is why... your employees are not assembly line workers. And a lot of what they do is very similar, but if you try to force people to standardize. If you try, then they will be like... no, I'll do it my way". (Manager, 2E)

Other meaningful work experiences relate to 
pursuing a purpose, intrinsic motivation and heuristic motivation. Across the board, employees were developing and improving organizational services to better fulfil their obligation to customers. Specifically, employee $3 \mathrm{C}$ found a way of justifying the value of her work by integrating the overall mission of the organization with her own intrinsic motivation. For example, she emphasized the importance of human interaction and improving the opportunities for providing public service:

"After all we work with humans. There is something about the contact that I, as a point of contact, have with the citizens, who have questions. There need to be some kind of ... link. [...] Explaining how the systems work, why things are the way they are, how the regulations and legislation work and such..." (Employee, 3C).

Furthermore, the organizational purpose of serving the public was found to align with her belief in the public system. She would take on a dual role of developing stronger human-centric service skills and become a controller of the system:

"You can't completely let it [the automated process] go either, at least not when it comes to what I do. Because, after all, it's other peoples' money that we manage. There must be a certain human control, I think" (Employee, 3C).

The alignment of organizational and personal values, and the opportunity to carry out this purpose through her work provides a clear person-organization fit, which positively influence the experience of work meaningfulness [17].

The problem of justifying the value of one's work is also known as anomie; a factor negatively influencing work meaningfulness. In organization 1, employees felt increasingly challenged to justify the value of their work, as previous work tasks were taken over by RPA. As an example, employee 1B experienced that there were no new tasks replacing tasks that was automated, which led her to question her own role in the organization. These are profound and significant human feelings, which neither serves the org. nor individual well. It may serve as a reminder of how deep automation of work with RPA can influence employees' feelings of self-worth.

\section{Discussion}

We found that all case organizations to a varying degree had awareness of the possibilities for creating meaningful work and pursuing societal value [14]. We also found that they did express their intentions and took initiatives with the potential to contribute to employees' experiences of work meaningfulness. However, neither of the companies did explicitly start their automation journey with the goal of creating more meaningful work. They rather followed more traditional parameters based on technological capabilities and existing key performance indicators, such as those mentioned in Syed et al., Güner et al. and Ivančić et al. [12][4][5]. This is in line with Vitharanage et al.'s observation and categorization of several benefits, such as increased human knowledge, skills and job satisfaction, which are consistent with experiences of work meaningfulness. However, these benefits are unintended and unexpected outcomes for both the organizations that automated their routine work and researchers who investigated their automation journey [6]. Consistent with our findings, Sampath and Khargonekar argue that organizations should not simply focus on cost- and productivity-driven automation objectives, but develop a holistic approach that promotes human well-being, moving towards socially responsible automation [14]. We found that case organization 2, whose employees are highly educated and performing more complex job tasks, were good at realizing these types of benefits. For example, by providing a higher degree of autonomy for their employees. They allowed their employees to focus on more complex work for the benefit of the employees and the organization as well. Organizations with similar characteristics as organization 2 may have a better potential for realizing these types of benefits. However, we argue that most organizations could benefit from conceptualizing meaningfulness when automating routine work and use work meaningfulness as an objective.

We found that automation of routine work with RPA may positively or negatively influence the opportunities for job crafting in the organizations. Opportunities for job crafting can be realized when employees experience that automation have freed up time from their usual work tasks. Employees can use this time to shape their jobs, engage in internal and external collaborations and working relationships, redevelop services, and/or thinking about and solving more complex work-related problems, which can result in value-adding solutions and innovations. Opportunities for job crafting can decline when employees experience that automation leads to more routine work, hence creating a feeling of alienation [19]. We found these opportunities to be influenced by amongst other the organizational structure, management and culture. According to Lysova et al.: "People are able to create meaningfulness when they have well-designed jobs with sufficient quality, type, and opportunities for job crafting that are embedded within organizations with facilitative leaders and cultures and a broader society that enables 
access to decent work"[10, p. 383]. In our findings, we found case organization 2 to be an exponent for a relatively successful approach where automation enabled new opportunities for job crafting in the accounting unit. As it were, the accountants managed to realize these opportunities by complementing their skills and experience with learning and applying new skills. However, we also found examples where automation did not further employees' experiences of meaningful work. For example, in case organization 1 , which is a highly compartmentalized organization, there is not a culture for for promoting employees' personal agency in performing their work. Hence, employees' opportunities for job crafting were found to be limited but occasionally possible for example in cross-departmental collaborations. The opportunity for job crafting, which is enabled by autonomy and personal agency, is considered highly significant for employees' possibilities to experience work meaningfulness [10, 16]. We argue that organizations with pre-defined job boundaries and roles (i.e. little autonomy, personal agency, thus limited opportunity for job crafting) have an obligation to take responsibility to facilitate employees' opportunities for achieving work meaningfulness.

In general, we found that automation of routine work with RPA had a profound influence on employees' experiences of meaningful work. A part of the reason could be that RPA as a technology differs from other ways of automating work, such as BPA, it typically involves business units to a greater extent [1]. Because managers and employees in the business units are better suited to make process-related decisions, we found employees in all case organizations to be involved in the process of mapping, standardizing, and operating the RPA robot. The involvement of employees with process expertise provides new opportunities for organizations to both develop their automation initiatives and facilitate meaningful work. For example, in case organization 3 where employees used their process expertise to both standardize and optimize the processes prior to automation. Employees, who make a significant contribution, could experience that they contribute to the purpose of the organization, which positively influence their perceived worthiness and experienced meaningfulness of work [17, 16, 19]. In case organization 2, employees used the opportunities of the automation initiative to create structural changes to the organization. Employees took initiative to complement their accounting skills with programming skills and seize the overall responsibilities for RPA in the accounting unit. In turn, their initiative was successful enough to cause structural changes in case organization
2 through the establishment of new, independent units. Employees in case organizations 2 and 3 used their expertise to identify new opportunities for automation and innovation [6], which helped them to create meaningful work experiences. This provides a good example of what Sampath and Khargonekar [14] refer to as steps towards socially responsible automation. In organizations with more human-centred approaches to automation, where they focus on automating work to leverage on human skills (i.e. augmenting human capabilities), automation was found to result in more enriching work, which is more likely to create meaningful work experiences. How and to what degree RPA cause changes in work and at work and facilitate and enable meaningful work for employees thus seem to be related to the organizations' structure, sector and approach to automation.

\section{Conclusion}

In this study we investigate how automation of routine work with RPA influence employees' experiences of work meaningfulness. We find that automation influences employees' experiences of work meaningfulness profoundly with positive and negative outcomes. Automation of routine work with RPA is a strong enabler for developing meaningful work as it (1) is often driven by employees with process expertise in the business units, who have autonomy and influence on the process. (2) The tasks that are automated are often tedious and time consuming, hence they liberate time for the employee. In most cases employees found this to result in more enriching work experiences, but in some cases it resulted in more routine work. (3) Where employees are afforded autonomy to design and craft their own jobs, they often found opportunities to develop their skill sets and/or engage with relevant others inside and outside their organization.

To the best of our knowledge, this study is the first study of automation of routine work with RPA, which focus on the employee perspective. This focus brings new opportunities for organizations to consider and conceptualize the value of their excising human resources. In the literature, the employee perspective appear to be under-appreciated and under-researched. Furthermore, to the best of our knowledge, we are the first to combine automation of work, RPA and work meaningfulness literature. Lastly, we provide rich empirical evidence of how automation of routine work can lead to employees' experiences of meaningful work from three organizations with offices in Oslo, Norway.

We believe that our study also has implications for practice. Organizations, who automate routine work 
with RPA, often focus on traditional organizational objectives such as improvement in accuracy, efficiency, and cost-reduction. Arguably, they do not have sufficient focus on the job level and employee level of the organization. Hence, they are not taking sufficient advantage of the human resources when automating work. Based on our findings, organizations serve employees and organizational interests best if they focus on creating autonomy for employees' and facilitate opportunities for job crafting.

In future research, we suggest focusing on further exploring the issue of automation of routine work with a meaningful work perspective. It is important to develop empirical research in different contexts and domains. We also encourage to study this issue with a longitudinal horizon and a learning perspective.

\section{References}

[1] M. C. Lacity and L. P. Willcocks, "Robotic process automation at telefónica O2," MIS Q. Executive, vol. 15, no. 1, 2016.

[2] J. Smids, S. Nyholm, and H. Berkers, "Robots in the workplace: a threat to-or opportunity for-meaningful work?," Philosophy Technology, 2019.

[3] M.-K. Stein, E. L. Wagner, P. Tierney, S. Newell, and R. D. Galliers, "Datification and the pursuit of meaningfulness in work," Journal of Management Studies, vol. 56, no. 3, pp. 685-717, 2019.

[4] L. Ivančić, D. Suša Vugec, and V. Bosilj Vukšić, "Robotic process automation: Systematic literature review," in Business Process Management: Blockchain and Central and Eastern Europe Forum, (Cham), pp. 280-295, Springer International Publishing, 2019.

[5] R. Syed, S. Suriadi, M. Adams, W. Bandara, S. J. Leemans, C. Ouyang, A. H. ter Hofstede, I. van de Weerd, M. T. Wynn, and H. A. Reijers, "Robotic process automation: Contemporary themes and challenges," Computers in Industry, vol. 115, p. 103162, 2020.

[6] D. Vitharanage, M. Imesha, W. Bandara, R. Syed, and D. Toman, "An empirically supported conceptualisation of robotic process automation (rpa) benefits," ECIS 2020 Research-in-Progress Papers, vol. 58, pp. 416 - 430, 2020.

[7] A. Shollo, M. Aanestad, K. Lyytinen, M. Mahring, P. A. Nielsen, and C. Sorensen, "Panel 4: A nordic sociotechnical perspective on artificial intelligence phenomena," in Proceedings of the 28th European Conference on Information Systems (ECIS), Proceedings of the European Conference on Information Systems, AIS Electronic Library (AISeL), 2020.

[8] S. Sarker, S. Chatterjee, X. Xiao, and A. Elbanna, "The sociotechnical axis of cohesion for the is discipline: Its historical legacy and its continued relevance," M I S Quarterly, vol. 43, pp. 695-719, Sept. 2019.

[9] M. Bergquist, H. Zinner Henriksen, A. Ojala, and X. Vassilakopoulou, "Sjis mission: Topical areas and research approaches," Scandinavian Journal of Information Systems, vol. 30, no. 2, pp. 3-4, 2018.

[10] E. I. Lysova, B. A. Allan, B. J. Dik, R. D. Duffy, and M. F. Steger, "Fostering meaningful work in organizations: A multi-level review and integration," Journal of Vocational Behavior, vol. 110, pp. 374 - 389, 2019. Review Issue 2017/18 (Annual issue).

[11] M. Schmitz, C. Stummer, and M. Gerke, "Smart automation as enabler of digitalization? a review of rpa/ai potential and barriers to its realization," Management for Professionals Future Telco, p. 349-358, 2018.

[12] E. O. Güner, S. Han, and G. Juell-Skielse, "Robotic process automation as routine capability: A literature review," In Proceedings of the 28th European Conference on Information Systems (ECIS), 2020.

[13] D. FERNANDEZ and A. AMAN, "Impacts of robotic process automation on global accounting services.," Asian Journal of Accounting Governance, vol. 9, pp. 123 $-131,2018$.

[14] M. Sampath and P. P. Khargonekar, "Socially responsible automation," Bridge, vol. 48, no. 4, pp. 45-72, 2018.

[15] G. Cheney, T. E. Z. Jr, S. Planalp, and D. J. Lair, "Meaningful work and personal/social well-being organizational communication engages the meanings of work," Annals of the International Communication Association, vol. 32, no. 1, pp. 137-185, 2008.

[16] B. D. Rosso, K. H. Dekas, and A. Wrzesniewski, "On the meaning of work: A theoretical integration and review," Research in Organizational Behavior, vol. 30, pp. 91 $127,2010$.

[17] C. Bailey, A. Madden, K. Alfes, A. Shantz, and E. Soane, "The mismanaged soul: Existential labor and the erosion of meaningful work," Human Resource Management Review, vol. 27, no. 3, pp. 416 - 430, 2017.

[18] J. R. Hackman and G. R. Oldham, "Development of the job diagnostic survey.," Journal of Applied Psychology, vol. 60, no. 2, pp. 159-170, 1975.

[19] D. A. Lepisto and M. G. Pratt, "Meaningful work as realization and justification: Toward a dual conceptualization," Organizational Psychology Review, vol. 7, no. 2, pp. 99-121, 2017.

[20] M. Lips-Wiersma, S. Wright, and B. Dik, "Meaningful work: differences among blue-, pink-, and white-collar occupations," Career Development International, vol. 21, pp. 534-551, Jan 2016.

[21] J. Lee, H. Lee, , and A. Suh, "Information technology and crafting of job: Shaping future of work?" PACIS 2018 Proceedings, p. 275, 2018.

[22] J. M. Corbin and A. Strauss, "Grounded theory research: Procedures, canons, and evaluative criteria," Qualitative Sociology, vol. 13, no. 1, p. 3-21, 1990.

[23] D. Courpasson and V. Monties, “"i am my body”. physical selves of police officers in a changing institution," Journal of Management Studies, vol. 54, no. 1, pp. 32-57, 2017.

[24] A. Marton, "Purposive selection and the quality of qualitative is research," in Proceedings of the 34th International Conference on Information Systems. ICIS 2013 (R. Baskerville and M. Chau, eds.), Association for Information Systems. AIS Electronic Library (AISeL), 2013. null ; Conference date: 15-12-2013 Through 18-12-2013.

[25] M. Myers, Qualitative Research in Business and Management. SAGE Publications, 2013.

[26] "Maxqda - all-in-one qualitative mixed methods data analysis tool," 2020. 